\title{
E-TRIP- Medication Total Points
}

National Cancer Institute

\section{Source}

National Cancer Institute. E-TRIP-Medication Total Points. NCI Thesaurus. Code C161760.

Emory T reatment Resistance Interview for PTSD (E-TRIP) Medication total points. 